\title{
Surgical Drill Guide for Insertion of an Infra-Acetabular Screw Based on an Anatomically Precontoured Plate System: A Cadaveric Study
}

\author{
Viola Freigang, ${ }^{1}$ Maximilian Gottsauner, ${ }^{2}$ Markus Rupp, ${ }^{1}$ Christian Pfeifer, ${ }^{1}$ \\ Stephan Grechenig, ${ }^{1}$ Alexander Kerner, ${ }^{3}$ Volker Alt, ${ }^{1}$ and Florian Baumann ${ }^{1}{ }^{1}$ \\ ${ }^{1}$ Department of Trauma Surgery, Regensburg University Medical Center, Germany \\ ${ }^{2}$ Department of Cranio-Maxillofascial Surgery, Regensburg University Medical Center, Germany \\ ${ }^{3}$ Division of Macroscopic and Clinical Anatomy, Graz, Austria \\ Correspondence should be addressed to Florian Baumann; florian.baumann@ukr.de
}

Received 6 May 2020; Accepted 3 July 2021; Published 27 July 2021

Academic Editor: Mattia Fortina

Copyright ( 2021 Viola Freigang et al. This is an open access article distributed under the Creative Commons Attribution License, which permits unrestricted use, distribution, and reproduction in any medium, provided the original work is properly cited.

\begin{abstract}
Purpose. Due to the anatomic structure of the pelvis, free-hand placement of screws in the acetabular fracture management can be difficult. Infra-acetabular screw fixation increases acetabular stability by distal fixation of the cup. Aim of this cadaveric study is to investigate if a plate-referenced drill guide can provide save placement of an infra-acetabular screw over a precontoured suprapectineal quadrilateral buttress plate (SQBP). Methods. We constructed a drill guide for an infra-acetabular screw based on the surface of an anatomically precontoured SQBP. A total of 12 adult cadaveric acetabular specimens were used for drill guide-assisted placement of the infra-acetabular screw. The drill guide contains a radiopaque spiral to allow longitudinal fine adjustment of the SQBP along the pelvic brim to assure correct position of the plate-drill-guide construct in relation to the Koehler's teardrop. After screw placement, we conducted a computed tomography (CT) scan of all specimens to assess the actual position of the screw in relation of the infra-acetabular corridor and the acetabular joint surface. Results. The position of the screw was within the infra-acetabular corridor in all cases. We did not see any intra-articular or intrapelvic screw penetration. The mean distance of the centerline of the screw to the medial border of the infra-acetabular corridor was $3.35 \mathrm{~mm}$. The secure distance to the virtual surface of the femoral head to was $7.3 \mathrm{~mm}$. Conclusions. A plate-referenced drill guide can provide safe placement of an infra-acetabular screw for treatment of acetabular fractures. Radiographic fine adjustment is necessary to access the optimal entry point.
\end{abstract}

\section{Introduction}

Due to the anatomic situation of the pelvis and acetabulum, management of an acetabular fracture is a technically demanding procedure. The close relationship to the iliac vessels and the complex construction of the acetabulum make it challenging to achieve reduction and fixation of the anterior and posterior acetabular column.

Demographic changes in industrial societies with overaging of the population have led to a shift in the typical fracture distribution. Large registry-based epidemiologic studies have demonstrated a rising number of acetabular fractures involving the anterior column over the last 20 years [1]. Lim- ited bone mineral density is a frequent epiphenomenon in geriatric acetabular fractures and reduces fixation possibilities. A dissociation of the anterior column to the posterior column followed by a medialization of the quadrilateral surface has been identified as a risk factor for a secondary dislocation leading to a so called "central subluxation" of the femoral head [2-4]. As a consequence, a number of advancements concerning anterior approaches and fixation techniques have been made recently [2,5-10]. Anatomically precontoured plate systems can provide enhanced reduction and fixation properties $[10,11]$. Plate systems with a buttress to support the quadrilateral surface provide increased periacetabular stability [11-14]. 


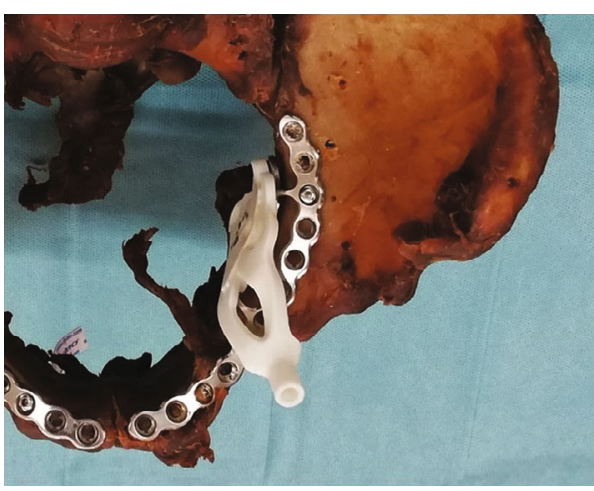

(a)

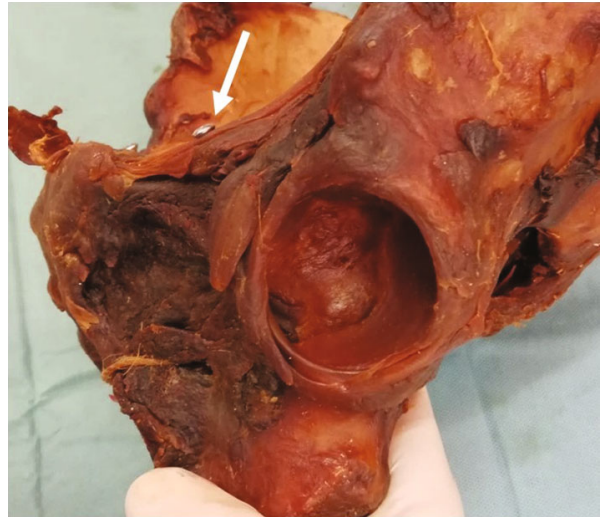

(b)

FIGURE 1: Fixation of the plate and drill guide construct to a left acetabulum (a) and image showing the infra-acetabular screw (white arrow) after removal of the plate (b).

The infra-acetabular screw following Letournel's [9, 12] concept of a periacetabular frame was reintroduced Culemann et al. in 2011 [13]. Marintshev et al. [14] proved increased stability with a screw inside the infra-acetabular corridor. Recently, a number of publications have investigated this bony corridor parallel to the quadrilateral surface centered in Koehler's teardrop [7, 15-17]. Since this corridor has a diameter of only few millimeters at level of the femoral head, the risk of an intraarticular screw placement for free-hand insertion is relatively high. A recently published radiomorphometric study revealed that the ideal entry point can be referenced to the tip of the iliopectineal eminence (IPE), and the drill angulation is remarkably constant in relation to the pelvic inlet plane (independent of age or gender) [15]. Anatomically precontoured suprapectineal plates show a convex bending reflecting the iliopectineal eminence. By matching the reference point (iliopectineal eminence) reflected by the convexity of the plate and the pelvic inlet plane (pectineal line) with the plate holes, a plate-referenced drill guide could ensure safe placement of an infra-acetabular screw.

The purpose of the study is to investigate if a platereferenced drill guide can provide save placement of an infra-acetabular screw over a precontoured suprapectineal quadrilateral buttress plate (SQBP).

\section{Materials and Methods}

A total of 12 adult cadaveric acetabular specimens (6 pelvises) preserved by Thiel's method [18] were used in the presented study. The embalming method preserves color, consistency, and biomechanical properties of the musculoskeletal tissue. None of the pelvises showed signs of abnormity like skeletal dysplasia or prior bony injury of the acetabulum.

The specimens were dissected and stripped of the surrounding soft tissue. The hip joint capsule was dissected at level of the capsule-labral junction (Figure 1).

2.1. Drill Guide Construction. We matched a 3D-CAD model of the anatomically precontoured plate to a mean 3D surface model of 516 pelvises (SOMA, Stryker Orthopaedic Model- ing and Analytics, Stryker Inc. Selzach, Switzerland). The shape of the surgical drill guide used in this study reflected the surface of the anatomically precontoured plate and had an optimal fit to the plate to assure a stable attachment of drill guide and plate. The drill guide was designed to contain the measurements determined in a recently published study on the ideal position of the infra-acetabular screw. Accordingly, the ideal entry point was $10.2 \mathrm{~mm}$ below and $10.4 \mathrm{~mm}$ medial to the highest point of the convexity of the plate reflecting the IPE. The angulation of the drill guide was in $10.4^{\circ}$ to the axial plane and $71.4^{\circ}$ to the sagittal plane of the pelvic inlet plane of the $3 \mathrm{D}$ model. The drill guide was constructed by additive manufacturing in a $3 \mathrm{D}$-printing process.

2.2. Surgical Technique. For referencing of the drill guide position, we used a suprapectineal quadrilateral buttress plate (SQBP) (PRO pelvis supra-pectineal plate, Stryker Inc., Selzach, Switzerland). The plate was placed aiming for an optimal fit of the anatomical precontoured shape and fixed starting with a parasymphysial screw and pressing the plate laterally to achieve an adequate fit on the pelvic brim to assure alignment with the pelvic inlet plane (Figure 1). Preliminary tests showed that this surgical routine-based proceeding missed required accuracy to access the optimal entry point of the infra-acetabular corridor. Fluoroscopic images revealed that there is somewhat longitudinal displaceability of the SQBP along the pelvic brim in direction of the linea terminalis.

Therefore, we added a radiopaque spiral to the drill guide to assure the correct position of the plate-drill-guide construct in relation to Koehler's teardrop (Figure 2). A fluoroscopic image in a combined obturator oblique outlet view was taken to assure overlap of the radiopaque spiral and Koehler's teardrop. Then, the plate was fixed by iliacal and pubic screws according to the surgical technique provided by the plate manufacturer (https://otaonline.org/ industry-partners/stryker/multimedia/16879679/pro-pelvissuprapectineal-plate-placement). After secure placement of the plate, we attached the drill guide to the plate and inserted another screw in the medial part of the plate (against the quadrilateral buttress) to secure the drill guide position and 


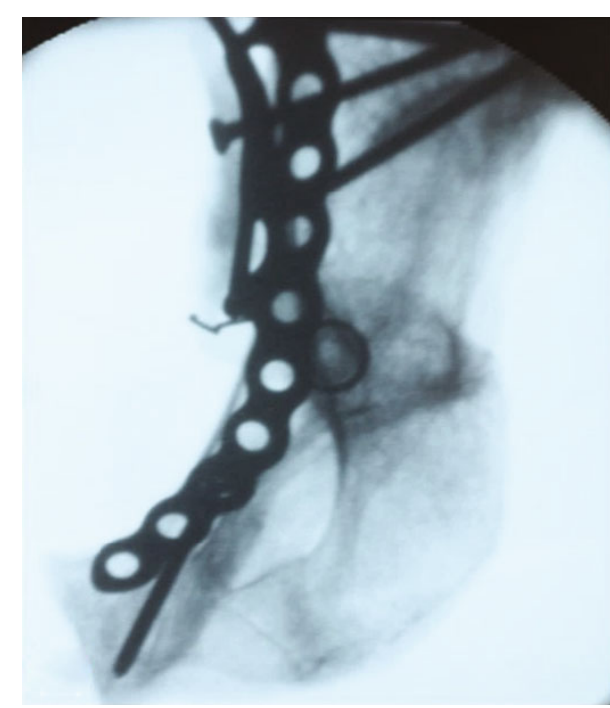

(a)

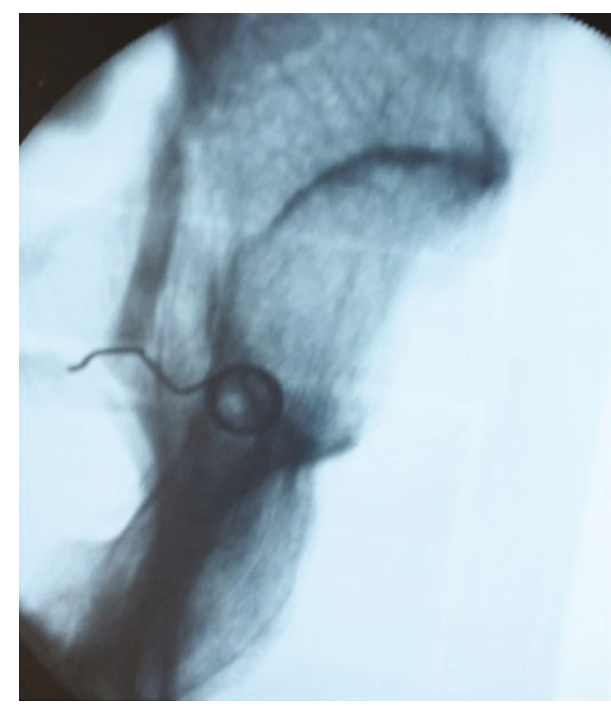

(b)

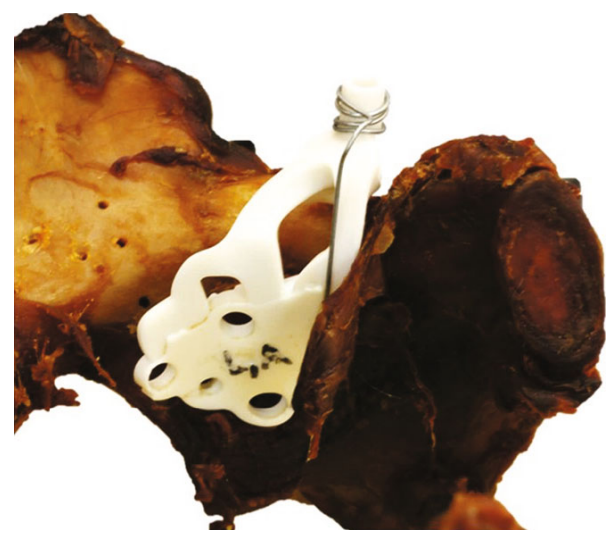

(c)

FIgURE 2: Fluoroscopy image for fine adjustment of the plate position (a) and an image showing the relation of Koehler's teardrop (center of the metal ring) and the drill guide (b) in a combined inlet oblique view along the infra-acetabular canal. Drill guide with a radiographic marker on a left acetabulum (c).

to avoid mediolateral tilting of the plate. After that, a $2.5 \mathrm{~mm}$ hole was drilled through the drill sleeve. The drill contained a depth measuring scale which was used to determine the length of the screw.

After insertion of the screw, the plate and the screws through the plate were removed leaving only the infraacetabular screw in place to reduce material artifacts of the computed tomography (CT) scan. Since the screw does not go through a plate hole, the plate could be removed without the risk of malplacement when reinserting the infraacetabular screw.

2.3. Radiographic Measurement. After drill guide-assisted placement of the infra-acetabular screw, we conducted a CT scan of all specimens to assess the actual position of the screw in relation of the infra-acetabular corridor and the acetabular joint surface. The position of the screw was measured using a picture archiving and communication system (PACS) software (Osirix 5.9, Pixmeo SARL, Bern, Switzerland). The infra-acetabular corridor was evaluated in a CT reconstruc- tion plane centered at level of the infra-acetabular corridor with an inlet angulation of $25^{\circ}$ according to Kanezaki and Miyazaki [16]. We characterized the infra-acetabular corridor by the diameter of the corridor perpendicular to the center of the femoral head center (IAD: infra-acetabular diameter), the distance of the center of the corridor to the virtual femoral head cartilage surface (CCD: center cartilage distance), and the length of the infra-acetabular corridor (IACL: infra-acetabular corridor length) measured by the screw length.

The measurement of the relation of the femoral head and the infra-acetabular screw was identified by generating a multiplanar reformation (MPR) centered through the core of the screw and the center of the femoral head according to Egli et al. [10] (Figure 3).

The distance of the center of the screw to the medial wall of the infra-acetabular corridor at level of the center of the femoral head (SCD: screw-corridor distance): To measure the distance of the center of the screw to the virtual cartilage surface of the femoral head (SFHD: screw femoral head 


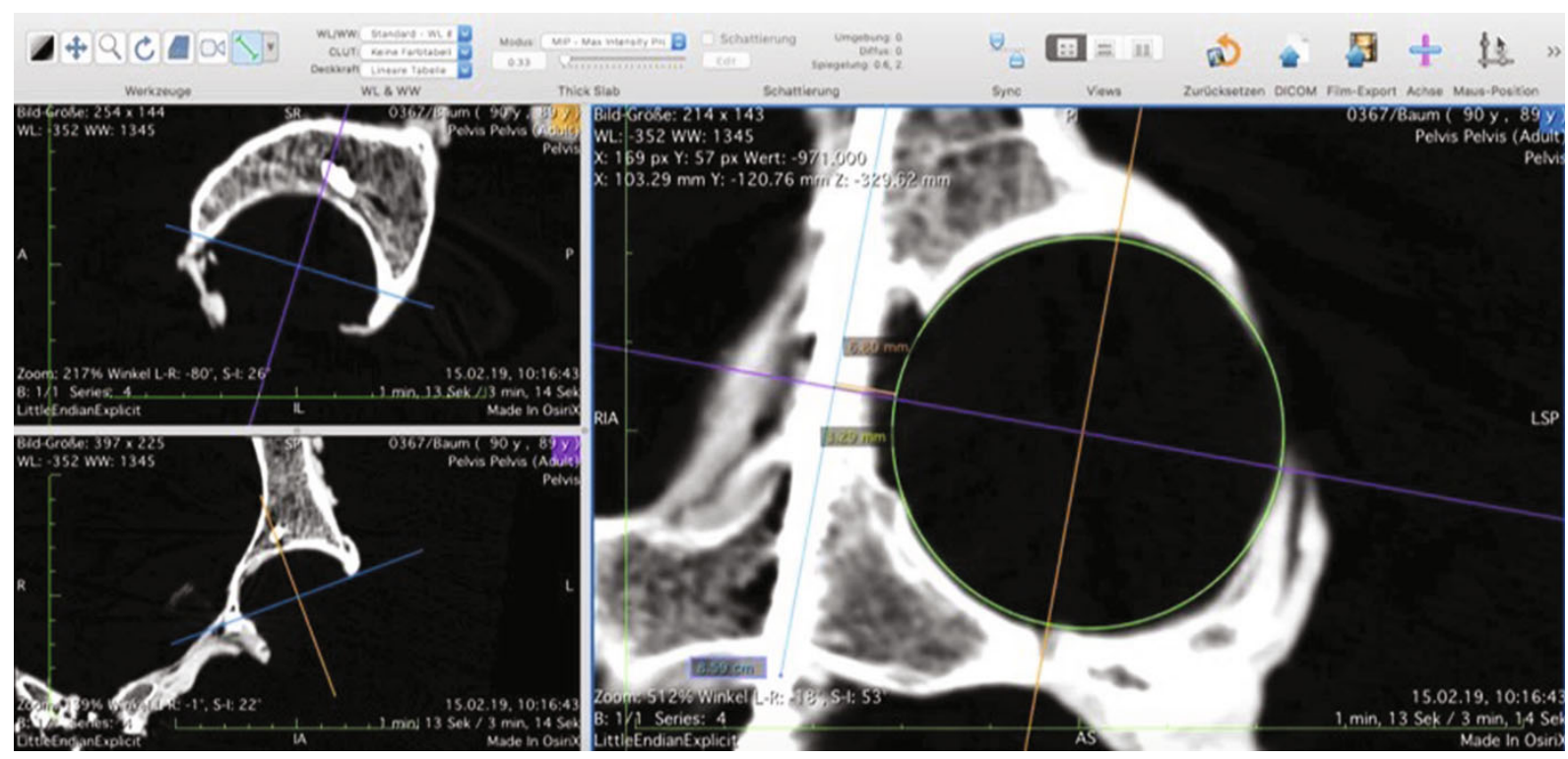

FIGURE 3: Screenshot illustrating software-based measurements of the CT-based MPR.

distance), we drew a virtual circle along the chondral border of the acetabulum to simulate the cartilage border of the head. All measurement results were recorded in $\mathrm{mm}$.

The cadaveric specimens were donated to the university anatomy program. Donors gave their informed written consent for the donation and anatomical dissection during lifetime. No further prehumous data like medical records were used in this study. Therefore, there was no necessity for an approval by an ethics committee.

2.4. Statistical Analysis. Statistical analysis was performed using the software package SPSS (Version 25, SPSS Inc, Chicago, Illinois). Since there is no previous data, this preliminary study was designed as an exploratory pilot study without any $a$ priori sample size calculation based on a primary endpoint. Based on the low variance of values in the previous radiomorphometric study and based on other experimental studies, a sample size of 12 specimens was considered feasible and expected to have enough power $[16,19]$. Unless otherwise stated, descriptive data are given as mean \pm standard deviation.

\section{Results}

The position of the screw was within the infra-acetabular corridor in all cases. We did not see any intra-articular or intrapelvic screw penetration. Table 1 shows measurement results recorded in 12 cadaveric acetabula. The mean diameter of the virtual femoral head was $45.1 \mathrm{~mm}( \pm 2.6)$. The infraacetabular corridor had a mean width (IAD) of $6.93 \mathrm{~mm}$ $( \pm 0.81)$ at level of the femoral head center. The mean distance of the middle of the corridor to the virtual cartilage surface of the femoral head (CCD) was $7.53 \mathrm{~mm}( \pm 1.42)$.

The mean corridor length measured at level of the screw (IACL) was $88.4 \mathrm{~mm}( \pm 7.4)$. The mean distance of the core of the screw to the medial wall of the infra-acetabular corridor (SCD) was $3.36 \mathrm{~mm}( \pm 0.83)$. The secure distance between screw and the virtual femoral head cartilage surface (SFHD) was $7.30 \mathrm{~mm}( \pm 1.71)$.

\section{Discussion}

This feasibility study demonstrated that a save placement of an infra-acetabular screw is possible by a plate-referenced drill guide over a precontoured SQBP. The entry point and angulation of the drill guide enabled save advancement of the drill directly into the infra-acetabular corridor. However, radiographic fine adjustment of the plate-drill-guide construct is indispensable to assure correct placement of the plate along the pelvic brim.

The secure distance between screw and the virtual femoral head cartilage surface was $7.30 \mathrm{~mm}$ (SFHD).

Demographic changes lead to an overaging of industrial societies and a rising number of geriatric fractures with a typical fracture pattern due to reduced bone quality in elderly $[1,3,20,21]$. Geriatric acetabular fractures typically involve the anterior column of the acetabulum. In the past, fractures involving the posterior wall or posterior column were much more frequent. The posterior Kocher/Langenbeck approach was the standard approach in treatment of fractures of the acetabulum [12, 22]. Within the past two decades, more and more geriatric fractures occur so that anterior approaches are used most frequent today $[1,8]$. This trend initiated further advancement of anterior approaches and operative techniques via anterior approaches [2, 4, 14, 23-27]. In 1993, Letournel was the first to describe a screw position through the acetabular fossa parallel to quadrilateral surface to increase intrinsic acetabular stability by a periacetabular frame construct [9].

Based on the quadrilateral screw described by Letournel [9], Culemann et al. [13] published a modified quadrilateral screw inserted more inferior in the region of Koehler's teardrop in 2011. This so-called "infra-acetabular" screw was intended to fix the anterior to the posterior column and to 
TABLE 1: Results of the computed tomography-based multiplanar reformation measurements given in $\mathrm{mm}$.

\begin{tabular}{|c|c|c|c|c|c|c|}
\hline $\begin{array}{l}\text { Specimen } \\
\text { No. } \\
\end{array}$ & $\begin{array}{c}\text { Infra-acetabular } \\
\text { diameter (IAD) }\end{array}$ & $\begin{array}{l}\text { Center cartillage } \\
\text { distance (CCD) }\end{array}$ & $\begin{array}{c}\text { Infra-acetabular } \\
\text { corridor length (IACL) }\end{array}$ & $\begin{array}{l}\text { Screw corridor } \\
\text { distance }(S C D)\end{array}$ & $\begin{array}{c}\text { Screw femoral head } \\
\text { distance (SFHD) }\end{array}$ & $\begin{array}{c}\text { Femoral head } \\
\text { diameter (FHD) }\end{array}$ \\
\hline$\overline{1 \mathrm{~L}}$ & 7.90 & 6.96 & 79.6 & 3.62 & 7.01 & 42.7 \\
\hline $1 \mathrm{R}$ & 8.06 & 7.12 & 80.7 & 3.24 & 6.52 & 42.3 \\
\hline $2 \mathrm{R}$ & 6.56 & 8.30 & 84.9 & 3.13 & 7.74 & 43.4 \\
\hline $2 \mathrm{~L}$ & 6.68 & 8.34 & 81.1 & 2.41 & 6.70 & 43.4 \\
\hline $3 R$ & 6.53 & 7.90 & 85.7 & 3.14 & 7.68 & 49.6 \\
\hline $3 \mathrm{~L}$ & 6.89 & 7.31 & 99.9 & 2.72 & 6.99 & 50.1 \\
\hline $4 \mathrm{R}$ & 5.83 & 6.36 & 99.8 & 2.98 & 6.72 & 43.0 \\
\hline $4 \mathrm{~L}$ & 7.08 & 7.39 & 85.9 & 3.29 & 6.80 & 42.8 \\
\hline $5 \mathrm{R}$ & 6.19 & 7.61 & 98.5 & 3.14 & 7.17 & 46.4 \\
\hline $5 \mathrm{~L}$ & 5.87 & 4.15 & 92.0 & 2.66 & 3.73 & 46.2 \\
\hline $6 \mathrm{R}$ & 8.12 & 9.30 & 85.9 & 4.95 & 9.98 & 45,2 \\
\hline $6 \mathrm{~L}$ & 7.44 & 9.57 & 86.9 & 5.05 & 10.50 & 45.8 \\
\hline Mean & 6.72 & 7.62 & 90.1 & 3.35 & 7.40 & 45.6 \\
\hline Range & 0.71 & 1.54 & 7.0 & 0.91 & 1.87 & 2.6 \\
\hline Min & 5.83 & 4.15 & 81.1 & 2.41 & 3.73 & 2.6 \\
\hline Max & 8.12 & 9.57 & 99.9 & 5.05 & 10.50 & 50.1 \\
\hline
\end{tabular}

minimize the risk of intraarticular penetration of the screw. A biomechanical study by Gras et al. [24] found that an infraacetabular screw significantly increases the stability of the acetabular fixation construct compared to a standard plate fixation. Standard implant size in management of acetabular fractures is a screw diameter of $3.5 \mathrm{~mm}$ requiring a drill hole for the infra-acetabular screw of $2.5 \mathrm{~mm}$. Drill guide-based drilling might provide enough accuracy for a secure placement of screws even in a narrow bony canal like the infraacetabular corridor.

In cases of reduced bone quality, a simple fall can cause a fracture of the anteromedial acetabulum or the quadrilateral surface by load transmission through the major trochanter [28]. A lack of support of the quadrilateral surface has been identified as a risk factor for a secondary dislocation leading to a so-called "central sub-luxation" of the femoral head $[4,20,21,24,29]$. In recent years, different concepts were introduced to increase medial support of the quadrilateral surface to prevent this complication $[2,4,13,14,23,24$, $27,28,30]$. Besides new plate designs like the SQBP and a screw placement close to the joint surface, the infraacetabular screw has increasingly attracted attention the discussion on advancements in acetabular fracture management $[10,16,31]$.

Intra-articular screw penetration can cause a cartilage damage of the femoral head. We did not see any intraarticular screw position. The CT measurements revealed a secure distance of the screw to the femoral cartilage surface of $7.30 \mathrm{~mm}$ (SFHD). The diameter of the infra-acetabular corridor at level of the femoral head center was $6.72 \mathrm{~mm}$.

Recently, a large biomorphometric CT-based study identified a viable infra-acetabular corridor with a diameter over $5 \mathrm{~mm}$ in $93 \%$ of specimens [7]. Due to variance of the pelvic morphology, referencing of the entry point to anatomic landmarks like the pubic symphysis is not accurate enough for clinical practice. Recently, a radiomorphometric study referenced the ideal entry point of the infra-acetabular screw $1 \mathrm{~cm}$ caudal and medial to the IPE in an angulation of $10^{\circ}$ in the axial plane and $70^{\circ}$ in the sagittal plane.

Instead of referencing the drill guide to the bony surface that might vary between each patient, the drill guide was referenced to a SQBP with a standardized surface. Using the plate as a reference, the interface between drill guide and plate allows an accurate fit without any deviation of the drill. Altering the level of patient-specific adjustment to the interface between plate and bone surface enlarges contact surface minimizing the risk of discrepancy of drilling direction in regard of the infra-acetabular corridor. In addition, conducting the drill process after insertion of the plate allows more room of action for the surgeon. The window of the intrapelvic approach provides a good overview and access to the anterior column and the quadrilateral surface. However, it can be difficult to manipulate with bulky instruments in the depth of the pelvis. Assembling the plate drill guide like a modular construct in situ might have an advantage over a large monobloc drill guide detecting the bone surface directly. The plate is constructed of annealed stainless steel which allows bending of the plate according to the bony surface. This implies the risk of mismatch of the drill guide and the plate when adaption by bending. His bending can also occur unintentionally by screw insertion and compression of the plate bone interface This has to be taken into account as a potential cause of mismatch of the plate-drill-guide interface and consequentially misguidance of the drill. However, most of the deflection of the plate takes place on the edges of the plate and the triangular shape of the plate at level of the drill guide prevents bending at this area. We did not observe any deflection of the plate at level of the platedrill-guide interface leading to a mismatch or deviation of the drill. There is also a trend towards personalized 
medicine. Several pilot studies have proven advantages of preoperative $3 \mathrm{D}$ model-based plate bending for acetabulum fractures [27, 32, 33]. Although this procedure is time consuming and costly, this may become the standard of care making a bending of the plate evitable.

There is an ongoing debate about "safe zones" and "unsafe zones" in acetabular fracture management [34]. The entry point of the infra-acetabular screw is located in an unsafe zone. Even with the knowledge of the ideal entry point, free-hand insertion implicates the risk of an intraarticular screw position. Therefore, a drill guide-based insertion could be rewarding to replicate the ideal drill hole for an infra-acetabular screw. The plate-referenced drill guide used in this study allowed save placement of an infra-acetabular screw over a precontoured SQBP in all cases. Further studies are necessary for clinical implementation.

This study has some limitations. We manufactured the drill guide based on the SQBP that is adapted to a mean pelvic model based on 516 pelvic 3D CT scans of healthy subjects. This construction might not be suitable for patients with extreme skeletal dysplasia or degeneration of the hip joint. However, the pelvises used in this cadaveric study were of geriatric subjects with degenerative changes with a deep acetabular fossa narrowing the infra-acetabular canal. Even in these cases, we did not record any intra-articular screw penetration. Another limitation is that the drill guide was applied in not fractured acetabular cadavers. Incongruence of the infra-acetabular corridor due to insufficient reduction in a fracture situation can lead to occlusion of the designated screw canal or discrepancy of the reference at level of the quadrilateral surface. The limited number of cadavers that we used in this study reduces generalizability. Intention of this preliminary pilot study was to prove the principle of SQBP-based drill guide screw placement and to gain practical experience.

Detection of longitudinal displaceability of the SQBP along the pelvic brim in direction of the linea terminalis was one of the major findings that can only be detected in practical application. Although we did not observe any deformation of the plate, this could lead to a mismatch of the plate and drill guide leading to deviation of the drill.

Further studies are necessary to proof reliability in a large number of cases and in clinical application.

\section{Conclusion}

A plate-referenced drill guide can provide safe placement of an infra-acetabular screw for treatment of acetabular fractures. Radiographic fine adjustment is necessary to access the optimal entry. The secure distance between screw and the virtual femoral head cartilage surface was $7.30 \mathrm{~mm}$ (SFHD). Further studies are needed to proof reliability in clinics.
Abbreviations
CCD: Center cartilage distance
CT: Computer tomography
IACL: Infra-acetabular corridor length

IAD: Infra-acetabular diameter

IPE: Iliopubic/Iliopectineal eminence

MPR: Multiplanar reformation

PACS: Picture archiving and communication software

SCD: Screw corridor distance

SFHD: Screw femoral head distance

SQBP: Suprapectineal quadrilateral buttress plate.

\section{Data Availability}

Please contact the author for data requests.

\section{Ethical Approval}

The cadaveric specimens were donated to the university anatomy program. No further prehumous data like medical records were used in this study. Therefore, there was no necessity for an approval by an ethics committee.

\section{Consent}

Donors gave their informed written consent for the donation and anatomical dissection during lifetime.

\section{Disclosure}

Level of evidence is the level V cadaveric study.

\section{Conflicts of Interest}

The authors declare that they have no conflicts of interest.

\section{References}

[1] B. G. Ochs, I. Marintschev, H. Hoyer et al., "Changes in the treatment of acetabular fractures over 15 years: analysis of 1266 cases treated by the German pelvic multicentre study group (DAO/DGU)," Injury, vol. 41, no. 8, pp. 839-851, 2010.

[2] C. A. Collinge and G. F. Lebus, "Techniques for reduction of the quadrilateral surface and dome impaction when using the anterior intrapelvic (modified Stoppa) approach," Journal of Orthopaedic Trauma, vol. 29, Supplement 1, pp. S20-S24, 2015.

[3] G. Tosounidis, U. Culemann, M. Bauer et al., "Acetabular fractures in the elderly. Outcome of open reduction and internal fixation," Der Unfallchirurg, vol. 114, no. 8, pp. 655-662, 2011.

[4] G. White, N. K. Kanakaris, O. Faour, J. A. Valverde, M. A. Martin, and P. V. Giannoudis, "Quadrilateral plate fractures of the acetabulum: an update," Injury, vol. 44, no. 2, pp. 159167, 2013.

[5] J. D. Cole and B. R. Bolhofner, "Acetabular Fracture Fixation Via a Modified Stoppa Limited Intrapelvic Approach Description of Operative Technique and Preliminary Treatment Results," Clinical Orthopaedics and Related Research, vol. 305, pp. 112-123, 1994.

[6] A. Gänsslen, S. T. Grechenig, M. Nerlich, M. Müller, and W. Grechenig, "Standard approaches to the acetabulum part 2: Ilioinguinal approach," Acta Chirurgiae Orthopaedicae et Traumatologiae Cechoslovaca, vol. 83, no. 4, pp. 217-222, 2016. 
[7] F. Gras, H. Gottschling, M. Schröder, I. Marintschev, N. Reimers, and R. Burgkart, "Sex-specific differences of the Infraacetabular corridor: a biomorphometric CT-based analysis on a database of 523 Pelves," Clinical Orthopaedics and Related Research, vol. 473, no. 1, pp. 361-369, 2015.

[8] E. Hirvensalo, J. Lindahl, and V. Kiljunen, "Modified and new approaches for pelvic and acetabular surgery," Injury, vol. 38, no. 4, pp. 431-441, 2007.

[9] E. Letournel, "The treatment of acetabular fractures through the ilioinguinal approach," Clinical Orthopaedics and Related Research, vol. 292, pp. 62-76, 1993.

[10] R. J. Egli, M. J. B. Keel, J. L. Cullmann, and J. D. Bastian, "Secure Screw Placement in Management of Acetabular Fractures Using the Suprapectineal Quadrilateral Buttress Plate," BioMed Research International, vol. 2017, 7 pages, 2017.

[11] T. Busuttil, M. Teuben, R. Pfeifer, P. Cinelli, H. C. Pape, and G. Osterhoff, "Screw fixation of ACPHT acetabular fractures offers sufficient biomechanical stability when compared to standard buttress plate fixation," BMC Musculoskeletal Disorders, vol. 20, no. 1, p. 39, 2019.

[12] R. Judet, J. Judet, and E. Letournel, "Fractures of the Acetabulum," The Journal of Bone and Joint Surgery. American Volume, vol. 46, no. 8, pp. 1615-1675, 1964.

[13] U. Culemann, I. Marintschev, F. Gras, and T. Pohlemann, "Infra-acetabular corridor-technical tip for an additional screw placement to increase the fixation strength of acetabular fractures," The Journal of Trauma, vol. 70, no. 1, pp. 244-246, 2011.

[14] I. Marintschev, F. Gras, C. E. Schwarz, T. Pohlemann, G. O. Hofmann, and U. Culemann, "Biomechanical comparison of different acetabular plate systems and constructs - The role of an infra-acetabular screw placement and use of locking plates," Injury, vol. 43, no. 4, pp. 470-474, 2012.

[15] F. Baumann, P. Schmitz, D. Mahr et al., "A guideline for placement of an infra-acetabular screw based on anatomic landmarks via an intra-pelvic approach," Journal of Orthopaedic Surgery and Research, vol. 13, no. 1, p. 77, 2018.

[16] S. Kanezaki, M. Miyazaki, N. Notani et al., "Analysis of computed tomography-based infra-acetabular morphometry to assess the feasibility of infra-acetabular screws," Archives of Orthopaedic and Trauma Surgery, vol. 140, no. 3, pp. 359364, 2020.

[17] S. Arlt, H. Noser, A. Wienke, F. Radetzki, G. O. Hofmann, and T. Mendel, "Secure corridor for infraacetabular screws in acetabular fracture fixation-a 3-D radiomorphometric analysis of 124 pelvic CT datasets," Journal of Orthopaedic Surgery and Research, vol. 13, no. 1, p. 119, 2018.

[18] W. Thiel, "an arterial substance for subsequent injection during the preservation of the whole corpse," Anatolia, vol. 174, no. 3, pp. 197-200, 1992.

[19] N. A. Alfonso, W. Ryan, T. Baldini, M. Reiter, C. Joyce, and C. Mauffrey, "Fixation of transverse acetabular fractures with Precontoured plates alone causes fracture malreduction: a biomechanical assessment," Journal of Orthopaedic Trauma, vol. 34, no. 2, pp. 89-94, 2020.

[20] T. A. Ferguson, R. Patel, M. Bhandari, and J. M. Matta, "Fractures of the acetabulum in patients aged 60 years and older: an epidemiological and radiological study," Journal of Bone and Joint Surgery. British Volume (London), vol. 92, no. 2, pp. 250-257, 2010.

[21] E. Pagenkopf, A. Grose, G. Partal, and D. L. Helfet, "Acetabular fractures in the elderly: treatment recommendations," HSS
Journal ${ }^{\circledR}$ : The Musculoskeletal Journal of Hospital for Special Surgery, vol. 2, no. 2, pp. 161-171, 2006.

[22] R. Judet, J. Judet, and A. Lanzetta, "Fractures of the acetabulum. Classification and guiding rules for open reduction," Archivio di Ortopedia, vol. 81, no. 3, pp. 119-158, 1968.

[23] A. Gänsslen, "Biomechanische Grundlagen bei osteoporotischer Frakturversorgung am Becken," Der Unfallchirurg, vol. 113 , no. 4, pp. 272-280, 2010.

[24] F. Gras, I. Marintschev, C. E. Schwarz, G. O. Hofmann, T. Pohlemann, and U. Culemann, "Screw- versus platefixation strength of acetabular anterior column fractures," Journal of Trauma and Acute Care Surgery, vol. 72, no. 6, pp. 1664-1670, 2012.

[25] G. Y. Laflamme, J. Hebert-Davies, D. Rouleau, B. Benoit, and S. Leduc, "Internal fixation of osteopenic acetabular fractures involving the quadrilateral plate," Injury, vol. 42, no. 10, pp. 1130-1134, 2011.

[26] A. Schäffler, S. Döbele, F. Stuby et al., "A new anatomical wing plate for osteoporotic acetabular fractures: biomechanical testing and first clinical experience," Zeitschrift für Orthopädie und Unfallchirurgie, vol. 152, no. 1, pp. 26-32, 2014.

[27] F. Gras, I. Marintschev, L. Grossterlinden et al., "The anterior intrapelvic approach for acetabular fractures using approach-specific instruments and an anatomical-preshaped 3-dimensional suprapectineal plate," Journal of Orthopaedic Trauma, vol. 31, no. 7, pp. e210-e216, 2017.

[28] A. Schäffler, S. Döbele, and F. Stuby, "A new anatomical wing plate for osteoporotic acetabular fractures: Biomechanical testing and first clinical experience," Z Orthop Unfall, vol. 152, no. 1, pp. 26-32, 2014.

[29] D. O. Verbeek, J. P. van der List, C. M. Tissue, and D. L. Helfet, "Long-term patient reported outcomes following acetabular fracture fixation," Injury, vol. 49, no. 6, pp. 1131-1136, 2018.

[30] U. Culemann, J. H. Holstein, D. Köhler et al., "Different stabilisation techniques for typical acetabular fractures in the elderly-a biomechanical assessment," Injury, vol. 41, no. 4, pp. 405-410, 2010.

[31] B. J. Kistler, I. R. Smithson, S. A. Cooper et al., "Are quadrilateral surface buttress plates comparable to traditional forms of transverse acetabular fracture fixation?," Clinical Orthopaedics and Related Research, vol. 472, no. 11, pp. 3353-3361, 2014.

[32] M. Boudissa, A. Courvoisier, M. Chabanas, and J. Tonetti, "Computer assisted surgery in preoperative planning of acetabular fracture surgery: state of the art," Expert Review of Medical Devices, 2018.

[33] S. Weidert, S. Andress, C. Linhart et al., "3D printing method for next-day acetabular fracture surgery using a surface filtering pipeline: feasibility and 1-year clinical results," International Journal of Computer Assisted Radiology and Surgery, vol. 15, no. 3, pp. 565-575, 2020.

[34] S. Zhang, W. Su, Q. Luo, F. Leung, and B. Chen, "Measurement of the Safe zone and the dangerous zone for the screw placement on the quadrilateral surface in the treatment of pelvic and acetabular fractures with Stoppa approach by computational 3D technology," BioMed Research International, vol. 2014, Article ID 386950, 2014. 